\title{
Synthesis and Characterization of Cellulose Nanofibers From Coconut Coir Fibers
}

\author{
Velayudham Navaneetha Krishnan and Atmakuru Ramesh* \\ Department of Analytical Chemistry, International Institute of Bio-technology and Toxicology (IIBAT), \\ Padappai, Chennai 601 301, Tamil Nadu, India.
}

\begin{abstract}
Cellulose nanofibers were isolated from coconut coir fibers by chemical treatment using alkaline, mineral acids and inorganic salts, followed by mechanical treatment and disintegration methods like sonication, cryo crushing and dissolution. The size and morphology of cellulose nanofibers were investigated by using the Field Emission Scanning Electron Microscope (FESEM). The width of synthesized cellulose nanofibers investigated by the FESEM was around $30 \mathrm{~nm}$ to $90 \mathrm{~nm}$ and few microns in length. Elemental analysis of cellulose nano fibers were confirmed with the Energy Dispersive Analysis (EDS) results. XRD study was conducted for the crystalline property of cellulose nanofibers synthesized from coconut coir fibers using standard microcrystalline cellulose as reference. FT-IR spectra confirmed the presence of hydroxyl groups, $C-H$ bond and the C-O-C groups in the synthesized cellulose nanofibers. The cellulose nano fibers were successfully utilized in the preparation of transparent thin film, filtration and water treatment.

Key words: Coconut coir fiber, Cellulose nanofibers, nanomaterial, microcrystalline
\end{abstract}

\section{Introduction}

Cellulose is made up with a number of glucose units linked by $\beta(1 \rightarrow 4)$ glycosidic linkage in a long chain. Hence, its properties are directly related to the chain length. One of the properties of cellulose is water insoluble, due to its glycosidic linkage between the monomers. The size reductions results in the fabrication of new material which is very easy for environmental degradation. The growth of bio composite materials with the natural fibers has become known in the most recent decade. This is one of the main advantages given by the wide accessibility of fibers, their low cost, weight and biodegradability. Coconut is widely cultivated in most of the countries. According to the Faostat (FAO 2008), the annual production of coconut in India, Brazil, Indonesia and Philippines is more than three million tons ${ }^{1}$. Due to this, the disposal of coconut waste is becoming a problem in the coconut producing countries. Alternatively, the usage of coconut coir fiber could be increased in the field of nanotechnology to minimize the environmental crash. The present investigation is to isolate cellulose nanofibers from coconut coir fiber for their vast application in environmental scavenging of waste material. Cellulose based biopolymers prepared from the cellulose rich materials were used in preparation of several biocomposite materials ${ }^{2-5}$. Cellulose has a strong affinity and hydrogen bonding through the Vander Val's force to itself, hence it is very stable and insoluble in water and most of the solvents. Cellulose can be dissolved, without derivation, in some hydrophilic ionic liquids, such as1-butyl-3-methyl imidazolium chloride (BMIMCl) and 1-allyl-3-methyl imidazolium chloride (AMIMCl), dimethylformamide, cadiumethylenediamine (cadoxen), cupriethylenediamine (CED) and N-methylmorpholine $\mathrm{N}$ - oxide ${ }^{6,7}$.

The physical and chemical properties of cellulose and its chemical reactivity and behavior are strongly influenced due to the arrangement of the hydroxyl group and hydrogen bonding with relation to each other and to the fiber axis. Native cellulose is arranged by sequences like amorphous region and crystalline region. When it undergoes chemical reaction, the amorphous region could get wrecked and the crystalline region isolated. Carboxylate cellulose nanocrystals are prepared from cotton linters by Tempo oxidation method ${ }^{8}$ Cellulose nanofibers are synthesized from wheat straw by chemical and mechanical treatment ${ }^{9}, 10$. The cellulose fibers have established great industrial applications, however the insufficient manufacturing technology hinder their commercial applications ${ }^{11}$. Cellulose, in its nano form, has great potential in emerging medical and environmental applications and it has proven to be a good heat transfer material ${ }^{12-14}$. Cellulose fiber has contributed in its use in paper and other fiber based composite materials in adsorbents, textile industry, cosmetics and medical fields. The electro-spinning process makes it possible to produce 50 to $500 \mathrm{~nm}^{15}$. The $\mathrm{HNO}_{3}-\mathrm{KClO}_{3}$ treatment is performed to produce biocomposites used from the bamboo plant ${ }^{16}$. The aim of this study is to prepare cellulose nanofibers from coconut coir fiber through the top down process. 


\section{Materials And Methods}

\section{a. Materials - Reagents and chemicals}

The coconut coir fibers were collected from local agriculture farm, near Padappai, Kanchipuram district, Tamil Nadu. Chemicals such as nitric acid, hydrochloric acid, sodium hydroxide, sodium hypochorite and liquid nitrogen were supplied from Merck specialties Pvt Ltd., India. These chemicals were pure and analytical grade chemicals, used directly without any purification.

A quantity of $2.5 \mathrm{~N}$ sodium hydroxide solution was prepared by weighing $50 \mathrm{~g}$ of sodium hydroxide into a $500 \mathrm{~mL}$ glass bottle and diluted with $500 \mathrm{~mL}$ distilled water. Another quantity of $2.5 \mathrm{~N} \mathrm{HCl}$ was prepared by adding $103.3 \mathrm{~mL}$ of concentrated $\mathrm{HCl}$ into a $500 \mathrm{~mL}$ volumetric flask and brought the volume up to the mark with distilled water. A quantity of $2.5 \mathrm{~N}$ Nitric acid solution was prepared by adding $78.8 \mathrm{~mL}$ of concentrated $\mathrm{HNO}_{3}$ into a $500 \mathrm{~mL}$ volumetric flask and brought the volume up to the mark with distilled water.

\section{b. Materials :- Instrumentation}

\subsubsection{Field Emission Scanning Electron Microscope}

The resulting cellulose material was investigated by FESEM Analysis (Model - Quanta FEG 200, FEI, and Netherlands). The operated accelerated maximum voltage is $30 \mathrm{KV}$ and the minimum is $200 \mathrm{~V}$, it has three modes, High Vacuum, Low Vacuum and Environmental SEM. The Cellulose size and its property were characterized by FESEM analysis at high resolution. The specimen was deposited on the electron microscope grids, operated at an acceleration voltage at $10 \mathrm{kv}$ with SE mode and observed.

\subsubsection{X-ray diffraction Analysis}

X-ray diffraction analysis helps to understand crystallinity and the amorphous nature of cellulose nanofibers. Microcrystalline cellulose sourced from Merck Specialities, Mumbai was used as reference for XRD characterization. The X-ray diffraction is a Panalytical XPert Pro XRD with both, powder mode and GI Attachments. The patterns are detected on XPert High Score Plus software.

\subsubsection{Energy dispersive X-ray Spectroscopy}

EDS is an analytical technique to identify the elements and elemental composition present in the sample. The EDS is a Liquid $\mathrm{N}_{2}$ based $30 \mathrm{~mm}$ detector EDAX model. The EDS analyses are integrated characteristics of the SEM.

\subsubsection{FT-IR spectroscopy}

The properties of the CCNF were characterized by FT-IR using a fourier transform infrared spectrophotometer, model-8400S, shimadzu. The analyses were carried out by the $\mathrm{KBr}$ pellet technique. The samples were prepared by mixing the 2 to $3 \mathrm{mg}$ of sample with approximately $250 \mathrm{mg}$ of $\mathrm{KBr}$ in to a mortar and ground well. The $\mathrm{KBr}$ pellets were prepared in a specified device under vacuum. The scanning range was from 400 to $4000 \mathrm{~cm}^{-1}$.

\section{c. METHODS}

\subsubsection{Preparation of Cellulose Nanofibers}

The schematic diagram of the preparation of cellulose fibers by different stages of treatments were illustrated below

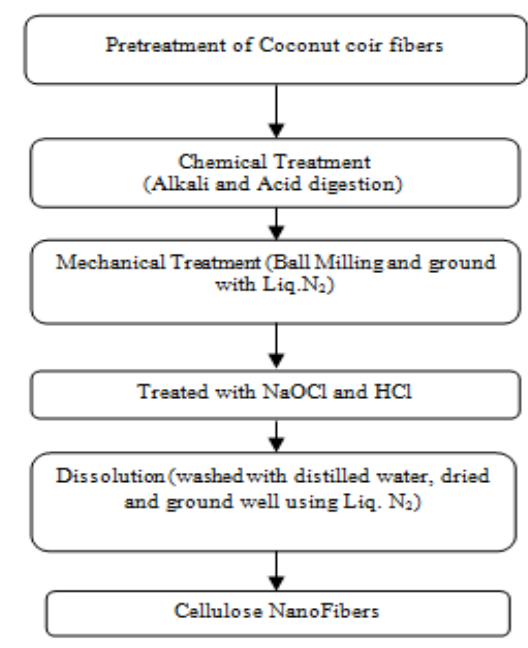




\subsubsection{Pretreatment}

Coconut coir fibers were separated and cut into small pieces and dried in sunlight, ground well using a homogenizer. The pulp was repeatedly washed with water and dried in a temperature controlled oven at $60^{\circ} \mathrm{C}$ for $18 \mathrm{~h}$. The pulp was transferred in to a soxhlet apparatus with a mixture of acetone and distilled water in a 1:1 ratio, to minimize the unwanted wood extractive. Around $20 \mathrm{~g}$ of dried pulp was weighed into a $250 \mathrm{~mL}$ round bottom flask and $125 \mathrm{~mL}$ of $2.5 \mathrm{~N}$ sodium hydroxide was added. The pulp was soaked in $2.5 \mathrm{~N}$ sodium hydroxide for $24 \mathrm{~h}$ at room temperature. Then the pulp was washed with plenty of distilled water, after which the same was soaked with $2.5 \mathrm{~N} \mathrm{HNO}_{3}$ acid for $48 \mathrm{~h}$. This was followed by digestion for $48 \mathrm{~h}$ at $60^{\circ} \mathrm{C}$ with $2.5 \mathrm{~N}$ sodium hydroxide. After the $\mathrm{NaOH}$ treatment, the pulp was washed well with plenty of water and digested using $2.5 \mathrm{~N}$ $\mathrm{HNO}_{3}$ at $60^{\circ} \mathrm{C}$ for $48 \mathrm{~h}$. The resulting pulp was washed with distilled water until the $\mathrm{pH}$ attained was neutral, and dried at $60^{\circ} \mathrm{C}$ in temperature controlled oven for $18 \mathrm{~h}$. In this step, most of the water soluble materials, lignin and pectin were removed, leaving behind the cellulose.

\subsubsection{Mechanical Treatment - Ball Milling}

Ball mill is used to grind the materials that are soft, medium hard to extremely hard, brittle or fibrous. The instrument has three modules, a grinding chamber (A), a grinding bowl (B) with balls and a threaded spindle with a three point grip (C). The grinding bowl is placed on a disc (D) inside the grinding chamber. The threaded spindle is placed firmly on the grinding bowl; it is made of agate material. The bowl is also provided with a removable lid $(\mathrm{G})$. The pretreatment pulp was milled by ball milling instrument (Model - Ball Mill PM 100, Make - Retsch Germany) with several times up to get a fine powder. After the Mechanical treatment, the resulting cellulose was characterized with Fluorescence microscope (Zeiss) and the images are presented.

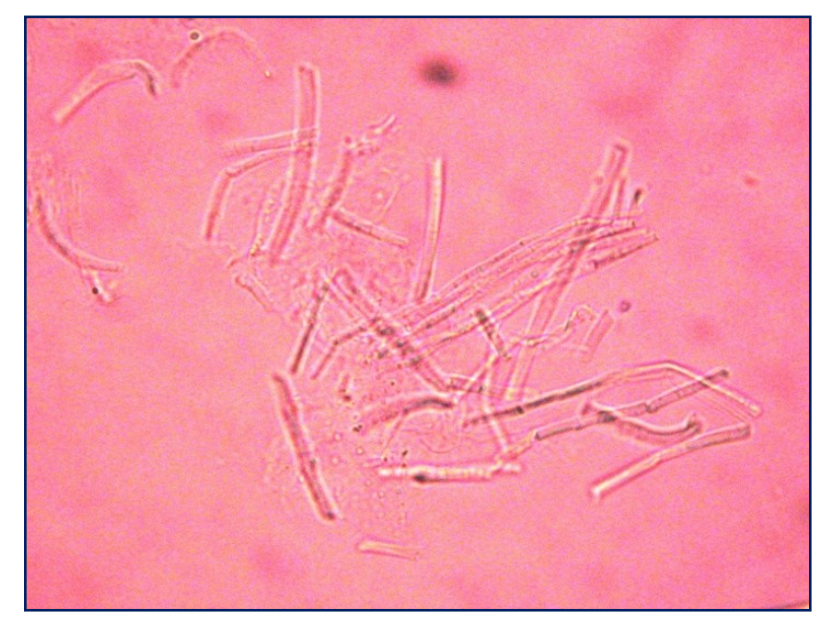

Figure 1 Microscope image for coconut coir fiber after mechanical treatment (ball milling)

The microscopic image of cellulose fiber obtained from coconut coir fiber (Figure 1) after the ball milling, the width was reduced from a centimeter to a few microns because of acid hydrolysis and mechanical treatment. The purity of cellulose fiber was increased due to the removal of lignin and conversion of hemicelluloses to cellulose.

\subsubsection{Chemical treatment with $\mathrm{NaOCl}$ and $\mathrm{HCl}$}

The pre-treated dried pulp was digested with the mixture of $10 \%$ wt sodium chlorite and $100 \mathrm{~mL}$ of $2.5 \mathrm{~N} \mathrm{HCl}$ under controlled temperature at $60^{\circ} \mathrm{C}$ for $24 \mathrm{~h}$ to break down the long chain molecule into cellulose unit. When the cellulose was treated with the mixture of sodium hypochlorite and hydrochloric acid, intermolecular hydrogen bonding broke down and the size of the chain was reduced. The resulting pulp was washed repeatedly with plenty of distilled water until the solution got to $\mathrm{pH}$ neutral and the pulp was dried in a temperature controlled oven at $50^{\circ} \mathrm{C}$ to dryness. The dried pulp was ground well by gradual addition of liquid nitrogen using a mortar in a fuming hood.

\subsubsection{Dissolution}

The cellulose fibers were mixed with $0.1 \mathrm{~N}$ sodium hydroxide solution, sonicated for $2 \mathrm{~h}$. Then the pulp was washed with distilled water by centrifugation and the $\mathrm{pH}$ maintained as neutral. Dried pulp was taken into a $100 \mathrm{~mL}$ stopper conical flask and $50 \mathrm{~mL}$ of distilled water was added and stirred with a magnetic pellet 
for $48 \mathrm{~h}$ and dried at $50^{\circ} \mathrm{C}$ up to dryness. The dried pulp was ground well by gradual addition of liquid nitrogen thrice. The resulting fiber was investigated for FESEM analysis and XRD.

\section{Result And Discussions}

The obtained FESEM image shows that the cellulose is in the form of fibers and the width of the range is $30 \mathrm{~nm}$ to $90 \mathrm{~nm}$. J. Zhang and his team have prepared cellulose nanomaterials in the form of spherical shaped particle ${ }^{17}$. The $\mathrm{HNO}_{3}-\mathrm{KClO}_{3}$ treatment followed by acid hydrolysis method cellulose nanocrystals was prepared from bamboo strands with the size of $50 \mathrm{~nm}$ to $100 \mathrm{~nm}^{18}$. FESEM image of CCNF obtained by coir fiber is represented in Figure 2.

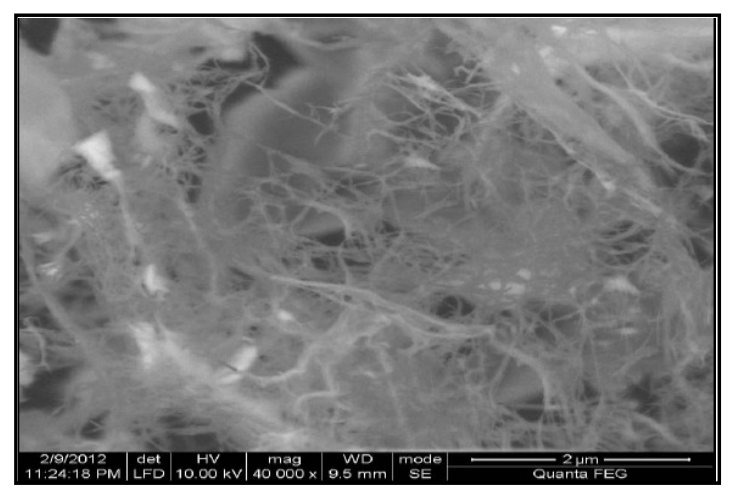

Figure 2. SEM image of the coconut coir cellulose Nanofibers

Figure 3 and Figure 4 show the XRD patterns of microcrystalline (reference) and coconut coir cellulose nanofiber respectively. From the microcrystalline cellulose XRD characteristics, a sharp peak is resolved which is indicative of crystalline in nature at $2 \theta=22.83^{\circ}$. The XRD characteristics of coconut coir cellulose nanofiber at $2 \theta=22.78^{\circ}$ and the peaks were broad which is indicative of amorphous nature. The peaks are broad on the nanomaterials, which indicate the presence of more of amorphous content in the nanomaterials. It could be more important due to having crystalline and amorphous regions; which will generate a more reactivity with the other chemicals to achieve the nano material functionality. The process of acetylation reduced the crystallinity of the fibers ${ }^{19}$. It was observed clearly that material obtained from coconut coir fiber has a partial amorphous and crystalline nature.

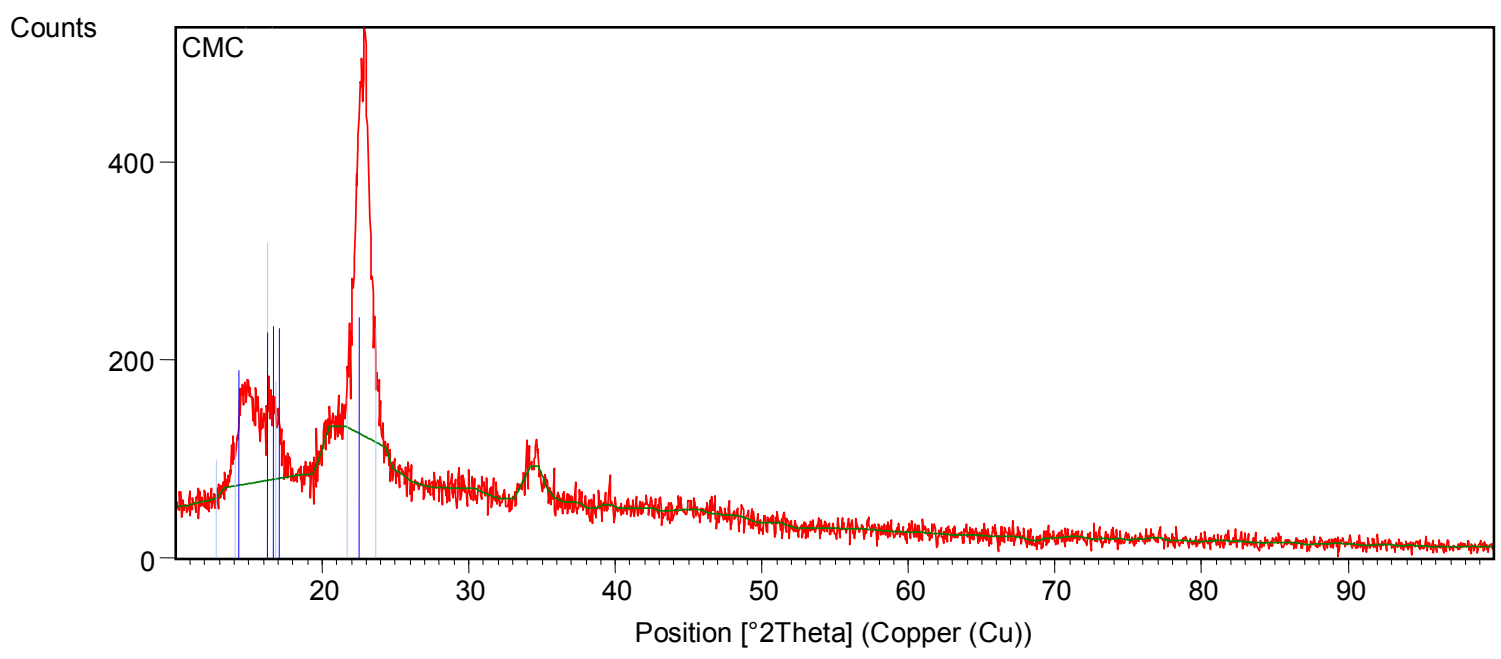

Figure 3. XRD Patterns of the cellulose microcrystalline 


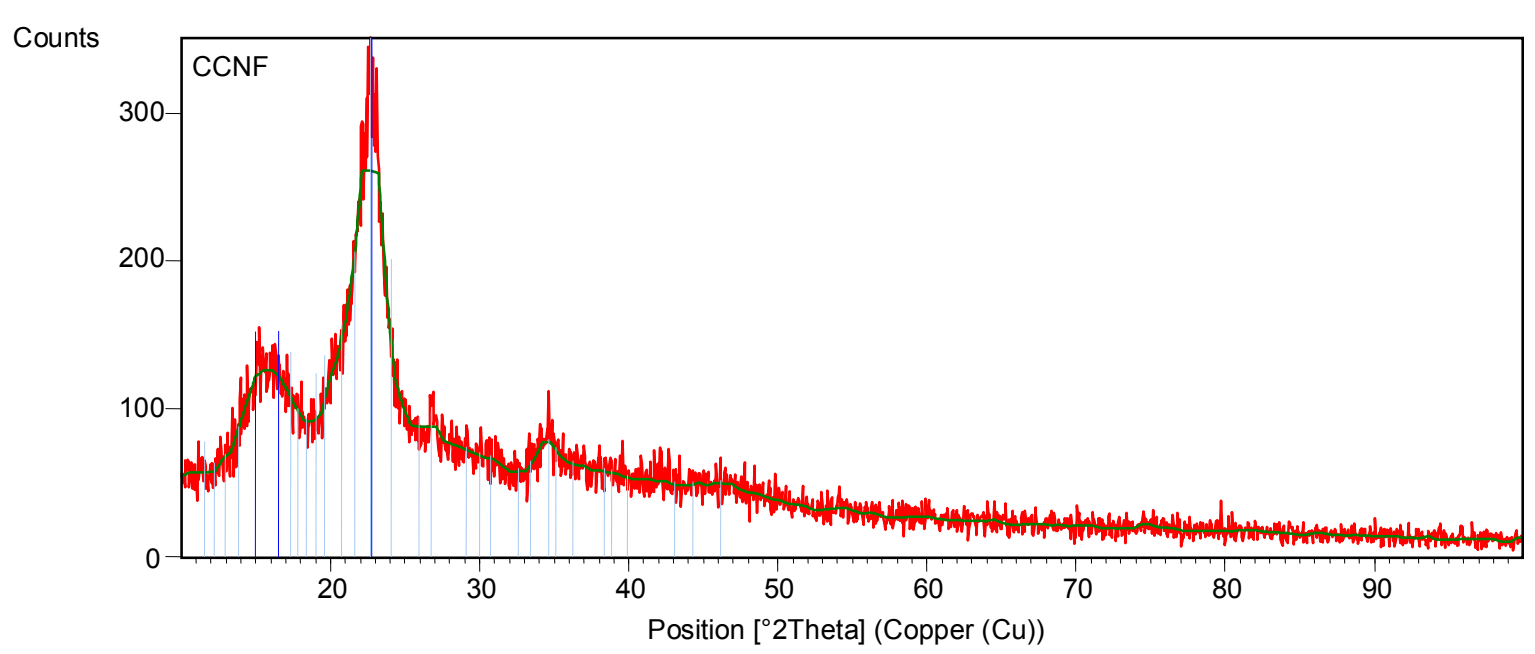

Figure 4. XRD Patterns of the coconut coir cellulose Nanofibers

Figure 5 represents the coconut coir cellulose nanofibers EDS spectrum. The EDS spectrum peaks of CCNF corresponding to the energy levels for which the carbon and oxygen. The comparison of carbon and oxygen percentage, the carbon percentage is higher than the oxygen percentage in the CCNF EDS spectrum. From this it can be concluded the carbon is likely slightly higher compared to oxygen in the CCNF. Elemental analysis of cellulose nano fibers were confirmed with the EDS results. The prepared cellulose nanofibers possess the skeleton of only carbon, oxygen and hydrogen. The chemicals associated with chemical treatment were not detected in the final cellulose Nanofibers, which has been confirmed by EDS analysis.

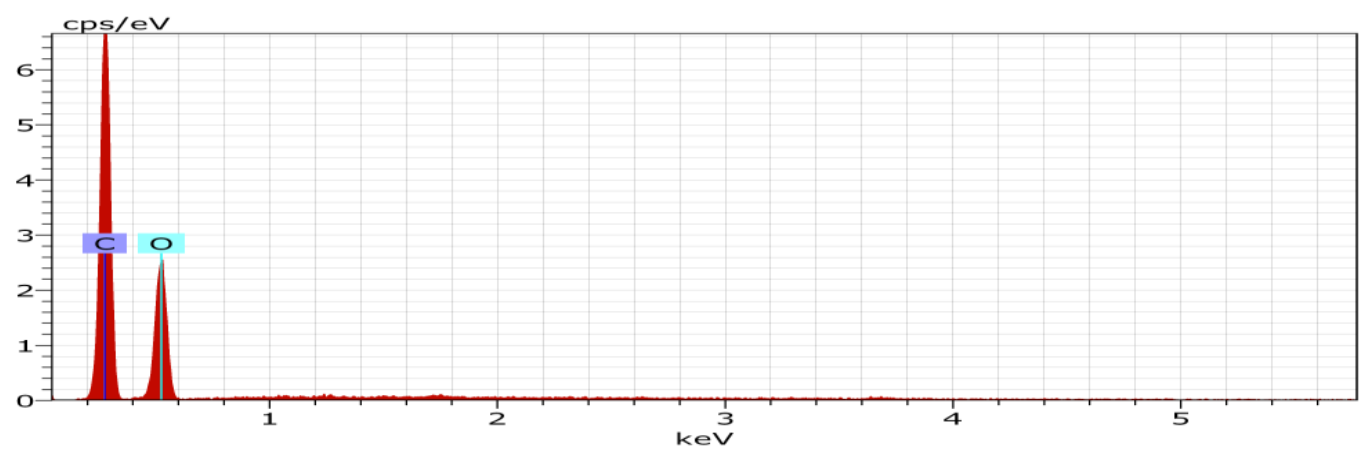

Figure 5 EDS spectrum of the coconut coir cellulose nanofiber

Figure 6 represents the FT-IR spectra for the CCNF respectively. The Strong dominant broad peaks in the region from 3700 to $3000 \mathrm{~cm}^{-1}$ are due to the stretching vibration of $-\mathrm{OH}$ groups. The $\mathrm{C}-\mathrm{H}$ stretching in methyl and methylene groups range between from 2800 to $3000 \mathrm{~cm}^{-1}$. The strong broad absorption peaks at $3354 \mathrm{~cm}^{-1}$ indicates the $-\mathrm{OH}$ stretching band (hydroxyl group), absorption peak at $2902 \mathrm{~cm}^{-1}$ indicates the aliphatic C-H stretching band, absorption peak at $1061 \mathrm{~cm}^{-1}$ indicates the C-O-C bending (ester functional group), the further absorption peaks at 1325 and $1371 \mathrm{~cm}^{-1}$ indicates the $\mathrm{C}-\mathrm{H}$ bending, absorption at $1433 \mathrm{~cm}^{-1}$ indicates the $\mathrm{CH}_{2}$ bending, absorption at $1163 \mathrm{~cm}^{-1}$ indicates the $-\mathrm{OH}$ bending. 


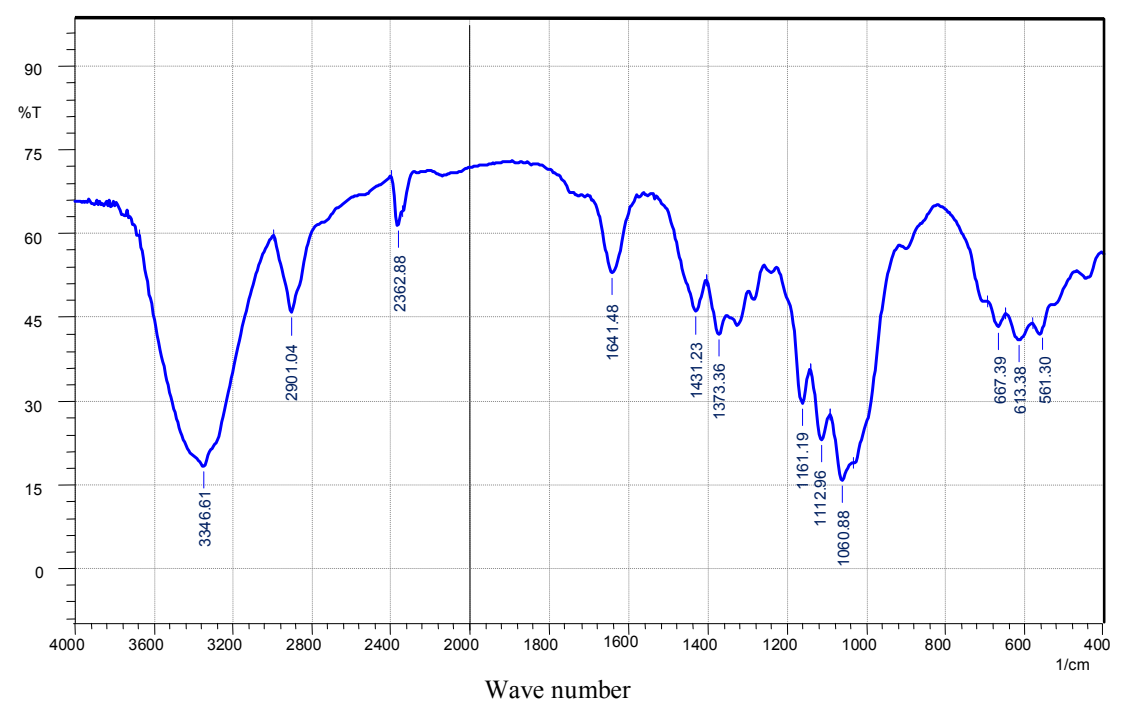

Figure 6 FTIR spectrum of the coconut coir cellulose nanofibers

\section{Conclusions}

This study is to demonstrate that the Cellulose Nanofibers can be synthesized from the coconut coir fiber through simple methods like pretreatment, mechanical treatment, chemical treatment followed by dissolution. The obtained cellulose nanofibers size and properties were characterized by SEM, XRD, EDS and FTIR analysis. The result shows that the synthesized nanofiber diameters are within range, between $30 \mathrm{~nm}-90$ $\mathrm{nm}$ with the length of few micron sizes. The results indicate that the cellulose nanofiber synthesized from coconut coir fiber can be useful in preparation of thin flims, absorbents, membranes, biosensors, biocomposites, protective clothing and biomedical application.

\section{Acknowledgements}

The authors are thankful to the management of IIBAT for valuable support and encouragement.

\section{References}

[1]. M. A. Esmeraldo, A. C. H. Barreto, J. E. B. Freitas, P. B. A. Fechine, A.S.B. Sombra, E. Corradini. G. Mele, A. Maffezzoli and S. E. Mazetto. BioResouces 5(4), 2478 - 2501 (2010)

[2]. W. A. MacDonald, J.Mater.Chem.14, 4 (2004)

[3]. S. R. Forrest, Nature. 428,911 ( 2004)

[4]. R .H. Reuss, B. R. Chalamala, A. Moussessian, M. G. Kane, A. Kumar, D. C. Zhang, J. A. Rogers, M. Hatalis, D. Temple, G. Moddel, B. J. Eliasson, M. J. Estes, J. Kunze ,E. S. Handy, E. S. Harmon, D. B. Salzman J. M.Woodall, M. A. Alam, J. Y. Murthy, S. C. Jacobsen, M. Olivier, D. Markus P. M. Campbell and E. Snow, Proc.IEEE. 93, 1239 (2005)

[5]. M. Nogi and H. Yano, Adv.Mater. 20, 1849 (2008)

[6]. R. P. Swatloski, S. K. Spear, D. John, J. D. Holbrey and R. D. Rogers, J.Am.Chem.Soc. 124,4974-4975 (2002)

[7]. H. Zhang, J. Wu, J. Zhangand and J. S. He, Macromolecules. 38,8272-8277 (2005)

[8]. S. Montanari, M. Roumani, L. Heux and Michel R. Vignon. Macromolecules. 38,1665-1671 (2004)

[9]. Thygesen and A. Oddershede, Cellulose. 12, 563-576 (2005)

[10]. Sain and M. Panthapulakkal, Ind. Crops Prod. 23, 1-8 (2006)

[11]. Micheal T. Postek, A. Vladar, J. Dagata, N. Farkas, B. Ming and Theodore H. Wegner, 24, 296-308 (2006)

[12]. T. Nishino, I. Matsuda, K. Hirao, Macromolecules. 37, (2004)

[13]. M. Nogi, K. Handa, A. N. Nakagaito, H. Yano, Appl.Phys.Lett. 87, 243-110 (2005)

[14]. Y. Shimazaki, Y. Miyazaki, Y. Takezawa, M. Nogi, K. Abe, S. Ifuku and H. Yano, Biomacromolecules. 8, 2976 (2007)

[15]. D. J. Gardner, G. S. Oporto, R. Mills and M. A. S. Azzi Samir, Journal of adhesion science and technology. 22, 545-567 (2008)

[16]. P. stand and M. Andresen, Cellulose. 15, $35-45$ (2008)

[17]. J. Zhang, Thomas, J. Elder, Yunqioa Pu, Arthu J. Ragauskas, Carbohydrate Polymer. 607-611 (2007)

[18]. D. Liu, T. Zhong, P. R. Chang, K. Li and Q. Wu, biosource technology 101, 2529-2536 (2010)

[19]. Mehdi Jonoobi, Jalauddin Haru, cellulose, 17, 299 (2010) 